\title{
Analysis of overlapping genetic association in type 1 and type 2 diabetes
}

\author{
Jamie R. J. Inshaw ${ }^{1}$ (D) - Carlo Sidore $^{2}$ (D) - Francesco Cucca $^{2}$ (D) - M. Irina Stefana ${ }^{1}$ (D) - Daniel J. M. Crouch ${ }^{1}$. \\ Mark I. McCarthy ${ }^{3,4}$ (D) $\cdot$ Anubha Mahajan ${ }^{3,4}$ (D) John A. Todd ${ }^{1}$ (D)
}

Received: 18 June 2020 / Accepted: 8 January 2021 / Published online: 8 April 2021

(C) The Author(s) 2021

\begin{abstract}
Aims/hypothesis Given the potential shared aetiology between type 1 and type 2 diabetes, we aimed to identify any genetic regions associated with both diseases. For associations where there is a shared signal and the allele that increases risk to one disease also increases risk to the other, inference about shared aetiology could be made, with the potential to develop therapeutic strategies to treat or prevent both diseases simultaneously. Alternatively, if a genetic signal co-localises with divergent effect directions, it could provide valuable biological insight into how the association affects the two diseases differently.

Methods Using publicly available type 2 diabetes summary statistics from a genome-wide association study (GWAS) meta-analysis of European ancestry individuals (74,124 cases and 824,006 controls) and type 1 diabetes GWAS summary statistics from a meta-analysis of studies on individuals from the UK and Sardinia (7467 cases and 10,218 controls), we identified all regions of $0.5 \mathrm{Mb}$ that contained variants associated with both diseases (false discovery rate $<0.01$ ). In each region, we performed forward stepwise logistic regression to identify independent association signals, then examined co-localisation of each type 1 diabetes signal with each type 2 diabetes signal using coloc. Any association with a co-localisation posterior probability of $\geq 0.9$ was considered a genuine shared association with both diseases.

Results Of the 81 association signals from 42 genetic regions that showed association with both type 1 and type 2 diabetes, four association signals co-localised between both diseases (posterior probability $\geq 0.9$ ): (1) chromosome 16q23.1, near CTRB1/ $B C A R 1$, which has been previously identified; (2) chromosome 11p15.5, near the INS gene; (3) chromosome 4p16.3, near TMEM129 and (4) chromosome 1p31.3, near PGM1. In each of these regions, the effect of genetic variants on type 1 diabetes was in the opposite direction to the effect on type 2 diabetes. Use of additional datasets also supported the previously identified co-localisation on chromosome 9p24.2, near the GLIS3 gene, in this case with a concordant direction of effect.

Conclusions/interpretation Four of five association signals that co-localise between type 1 diabetes and type 2 diabetes are in opposite directions, suggesting a complex genetic relationship between the two diseases.
\end{abstract}

Keywords Analyses · Co-localisation · Genetics · Genome-wide association study · Insulin · Statistics · Systematic · Type 1 diabetes · Type 2 diabetes

Anubha Mahajan and John A. Todd contributed equally to the work.

Jamie R. J. Inshaw

jinshaw@well.ox.ac.uk

$\triangle$ John A. Todd

jatodd@well.ox.ac.uk

1 JDRF/Wellcome Diabetes and Inflammation Laboratory, Wellcome Centre for Human Genetics, Nuffield Department of Medicine, University of Oxford, Oxford, UK
2 Institute for Research in Genetics and Biomedicine (IRGB), Cagliari, Sardinia, Italy

3 Wellcome Centre for Human Genetics, Nuffield Department of Medicine, University of Oxford, Oxford, UK

4 Present address: Genentech, South San Francisco, CA, USA 


\section{Research in context}

\section{What is already known about this subject?}

- Other than insulin, there are currently no treatments for both type 1 and type 2 diabetes

- Findings that genetic variants near the GLIS3 gene increase risk of both type 1 and type 2 diabetes have indicated shared genetic mechanisms at the level of the pancreatic beta cell

\section{What is the key question?}

- By examining chromosome regions associated with both diseases, are there any more variants that affect risk of both diseases and could support common mechanisms and repositioning of therapeutics between the diseases?

\section{What are the new findings?}

- At current sample sizes, there is evidence that five genetic variants in different chromosome regions impact risk of developing both diseases

- However, four of these variants have the opposite direction of effect in type 1 diabetes compared with type 2 diabetes, with only one, near GLIS3, having a concordant direction of effect

\section{How might this impact on clinical practice in the foreseeable future?}

- Genetic findings have furthered research in type 1 and type 2 diabetes independently and have suggested therapeutic strategies. However, our current investigation into their shared genetics suggests that repositioning of current type 2 diabetes treatments into type 1 diabetes may not be straightforward

\begin{abstract}
Abbreviations
FDR False discovery rate

GWAS Genome-wide association study

H4PP Posterior probability of co-localisation

LD Linkage disequilibrium
\end{abstract}

\section{Introduction}

There is a genetic component to both type 1 and type 2 diabetes, with approximately 60 chromosome regions associated with type 1 diabetes [1] and over 200 associated with type 2 diabetes [2] at genome-wide significance. Examination of regions associated with both diseases could uncover signals that simultaneously alter disease risk for both diseases, termed colocalisation. Uncovering co-localising signals could provide biological insights into shared disease mechanisms, and potentially reveal therapeutic targets effective for both diseases. A recent analysis suggested that the same genetic variant alters risk of both type 1 and type 2 diabetes in five regions, near CENPW, CTRB1/BCAR1, GLIS3, BCL11A and THADA [3].

Here, we identified all regions across the genome that showed evidence of association with both type 1 and type 2 diseases at a false discovery rate (FDR) $<0.01$ and assessed colocalisation between the two diseases in each of these regions. Furthermore, to account for the possibility of multiple causal variants within an associated region, we extended the analysis to investigate conditionally independent associations within each region, to assess whether any of the associations with one disease co-localised with any associations in the other.

\section{Methods}

Type 1 diabetes meta-analysis summary statistics were generated using genome-wide association study (GWAS) data from 3983 cases and 3994 controls from the UK (genotyped using the Illumina Infinium 550K platform), 1926 cases and 3342 controls from the UK (genotyped using the Affymetrix GeneChip 500K platform) and 1558 cases and 2882 controls from Sardinia (genotyped using the Affymetrix 6.0 and Illumina Omni Express platforms), totalling 7467 cases and 10,218 controls (Electronic supplementary material [ESM] Table 1). Genotypes were imputed using the Haplotype Reference Consortium reference panel for the UK collections [4], and a custom Sardinian reference panel of 3514 Sardinians for the Sardinian collection (ESM, Imputation).

Summary statistics for type 2 diabetes were from 74,124 cases and 824,006 controls of European ancestry, imputed using the Haplotype Reference Consortium reference panel [2].

Regions associated with both diseases were identified by selecting all variants with type 1 diabetes and a type 2 diabetes association with an FDR $<0.01$ (ESM Methods, Type 1 diabetes GWAS). In each such region, windows of approximately $0.5 \mathrm{Mb}$ were taken to examine co-localisation (ESM Methods, Regions associated with both diseases). Within these regions, forward stepwise logistic regressions were carried out for both diseases, and conditional summary statistics were obtained so each conditionally independent signal from both diseases could be tested against each other for colocalisation (ESM Methods, Conditional analyses). 
Co-localisation of signals was assessed using coloc [5], a Bayesian method that enumerates the posterior probability that the association signals in a region are shared between traits. The prior probability of association with either disease was taken to be $1 \times 10^{-4}$ and the prior probability that the association signal is shared across traits was taken to be $5 \times 10^{-6}$, as recommended [6]. The threshold to consider signals as colocalising was conservatively chosen at a posterior probability $\geq 0.9$. Co-localisation was also examined using an alternative approach, as a secondary analysis, eCAVIAR [7] (ESM Methods, eCAVIAR).

Code used to carry out this analysis is available at https:// github.com/jinshaw16/t1d-t2d-colocalisation.

\section{Results}

Including conditionally independent association signals, 81 co-localisation analyses were carried out across 42 chromosomal regions that showed association with both diseases (ESM Table 2).

Four signals showed evidence of co-localisation using coloc, and these were also the regions with the highest eCAVIAR regional co-localisation posterior probabilities
(ESM Table 3). The first was on chromosome 16q23.1, near $C T R B 1$ and $B C A R 1$, with a posterior probability of colocalisation (H4PP hereafter) of 0.98 (ESM Fig. 1). The minor $\mathrm{A}$ allele at the type 2 diabetes index variant, rs 72802342 $(\mathrm{C}>\mathrm{A})$, is protective for type 2 diabetes (OR 0.87 , $p=4.00 \times 10^{-32}$ ) and susceptible for type 1 diabetes (OR 1.33 , $p=5.81 \times 10^{-10}$ ).

The second was on chromosome $11 \mathrm{p} 15.5$, near INS, where the primary type 2 diabetes association co-localised with the secondary type 1 diabetes association $(\mathrm{H} 4 \mathrm{PP}=0.95$, ESM Fig. $2)$. The direction of effect was opposite, with the minor $A$ allele at the type 2 diabetes index variant, rs4929965 (G>A), associated with susceptibility to type 2 diabetes (OR 1.07, $p=4.80 \times 10^{-25}$ ) and protection from type 1 diabetes (OR $0.87, p=1.89 \times 10^{-5}$ ).

Third, a region on chromosome $4 \mathrm{p} 16.3$ co-localised (H4PP=0.97) (Fig. 1), near TMEM129. The minor T allele at the type 2 diabetes index variant, rs56337234 (C>T), was associated with decreased risk of type 2 diabetes (OR 0.94, $p=1.4 \times 10^{-17}$ ) and increased risk of type 1 diabetes (OR 1.12, $p=4.07 \times 10^{-6}$ ).

Finally, a region on chromosome $1 \mathrm{p} 31.3$, near $P G M 1$, colocalised (H4PP=0.91, ESM Fig. 3), with the minor T allele at the type 2 diabetes index variant rs2269247 (C>T) decreasing
Fig. 1 Manhattan plots showing (a) gene locations and $-\log _{10} p$ value of association for each variant by position along chromosome 4 (genome build 37) in the TMEM129 region for (b) type 2 diabetes (T2D) and (c) type 1 diabetes (T1D), coloured by $r^{2}$ to the type 2 diabetes index variant, rs56337234 a

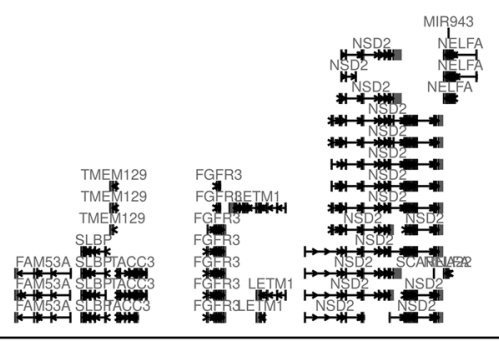

b

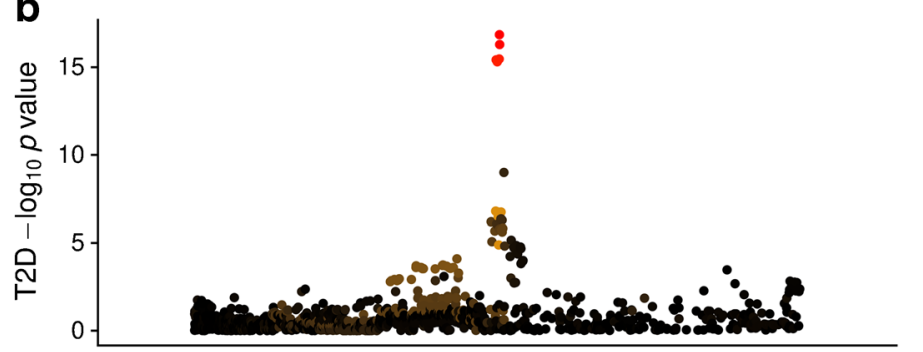

LD with T2D index variant 1.00 0.75 0.50 0.25

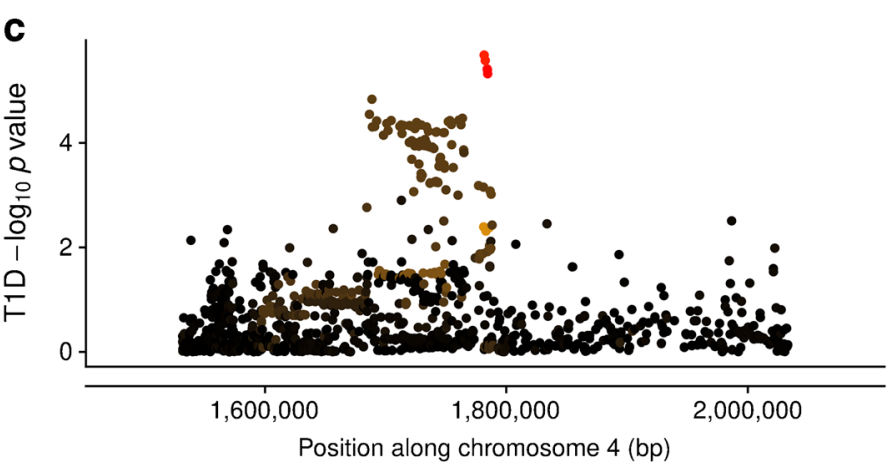

LD with T2D index variant 1.00 0.75 0.50 0.25 


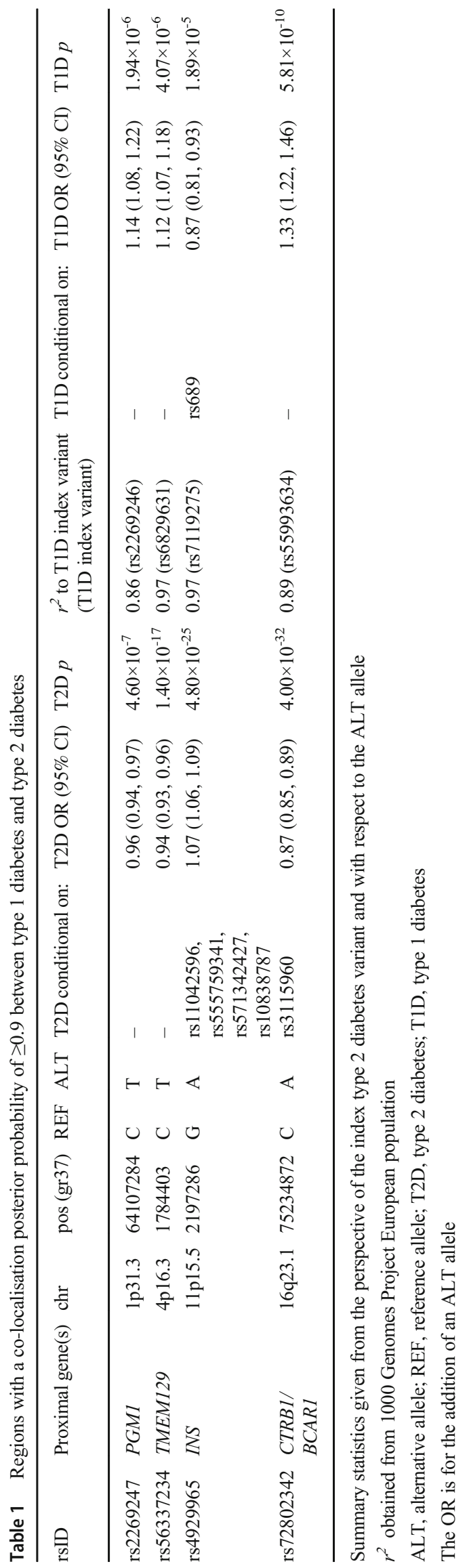


risk of type 2 diabetes (OR $\left.0.96, p=4.6 \times 10^{-7}\right)$ and increasing risk of type 1 diabetes (OR 1.15, $p=1.9 \times 10^{-6}$ ) (Table 1 ).

We did not replicate the finding that the chromosome regions near CENPW, GLIS3, BCL11A or THADA colocalised between type 1 and type 2 diabetes (H4PP $C E N P W=0.12, G L I S 3=0.29, B C L 11 A=0.28$, THADA not examined as no type 1 diabetes association existed in the region [FDR=0.07]). To investigate these discrepancies, we examined two other large type 2 diabetes meta-analyses: a trans-ethnic study including 1,407,282 individuals [8] and a study of 433,540 individuals of East Asian ancestry [9]. For the $C E N P W$ and $B C L 11 A$ regions, the type 2 diabetes signal is consistent with at least one of the other GWAS studies (measured by linkage disequilibrium [LD] in Europeans to the other study index variants, ESM Table 4), and the type 1 diabetes index variant is not in strong $\operatorname{LD}\left(r^{2}<0.41\right)$ with any of the index variants for type 2 diabetes across the three GWAS studies. However, at GLIS3, there appears to be a distinct signal in the European study [2] compared with the trans-ethnic and East Asian type 2 diabetes studies $\left(r^{2}=0.65\right)$, and the index variants from these two studies are in higher $r^{2}$ with the type 1 diabetes signal in our analysis $\left(r^{2}=0.68\right)$, and even higher $r^{2}$ with the index variant from a larger type 1 diabetes genetic analysis [1] $\left(r^{2}=0.99\right)$, indicating that the signal near GLIS3 does co-localise between type 1 and type 2 diabetes with concordant direction of effect, as previously identified [10].

\section{Discussion}

Using genetic association summary statistics from European populations, we identified 42 regions that showed association with both type 1 and type 2 diabetes, with 81 conditionally independent association signals across those regions. Four signals (near CTRB1/BCARI, INS, TMEM129 and PGM1) co-localised between the diseases, including a signal at the complex INS region for the first time, which was achieved by examining conditional summary statistics. However, in all four cases, the allele increasing risk for one disease was protective against the other. Examination of additional transethnic and East Asian type 2 diabetes genetic analyses indicated that a fifth association, near GLIS3, is likely to colocalise between diseases, with concordant direction of effect.

Given the distinct mechanisms underlying beta cell dysfunction and cell death between the two diseases [11], it is perhaps unsurprising that no additional signals were detected with concordant direction of effect. However, the type 1 diabetes GWAS was much smaller than the type 2 diabetes analysis, and therefore had less statistical power to detect more subtle genetic effects. If a type 1 diabetes GWAS were to be performed with similar power to the type 2 diabetes GWAS, more regions might co-localise between the two diseases, but either the effects of these additional regions on type 1 diabetes would be small compared with the currently known associations or they would be rare variants with larger effect sizes.

That four of five co-localisation signals had opposite directions of effect implies a complex genetic relationship between the two diseases. While the directional discordance offers little hope for effective treatments for both diseases simultaneously at these particular targets, it can offer biological insight into the disease pathways that these regions act upon, and even if there is directional discordance, the genetics could be highlighting the same therapeutic target.

We did not replicate the findings that the associations near BCL11A, CENPW and THADA co-localise between the two diseases [3], despite overlapping samples and similar numbers of cases and controls in the type 1 diabetes GWAS. There are three possible reasons for this: 1) the previous study [3] examined co-localisation using weaker association signals, for example, the co-localisation near THADA was based on a type 1 diabetes association $p$ value of $0.01 ; 2$ ) we used a more stringent prior for co-localisation between the two diseases, as recently suggested [6] $\left(5 \times 10^{-6}\right.$ vs $\left.1 \times 10^{-5}\right)$; and 3$)$ we used a more stringent posterior probability threshold to declare colocalisation ( 0.9 vs 0.5$)$. Our increased stringency compared with the previous analysis [3], while increasing the probability that any identified shared signals will be true positives, may have decreased our sensitivity to detect all co-localisations. For example, by examining other large type 2 diabetes GWAS analyses and a larger type 1 diabetes genetic analysis, we conclude that the association near GLIS3 likely does colocalise between the two diseases, and with concordant directions of effect.

In conclusion, with current GWAS sample sizes, just five associations appear to co-localise between type 1 diabetes and type 2 diabetes, four with opposing direction of effect. Larger sample sizes would be required to identify the depth of genetically identified therapeutic targets to treat or prevent both diseases simultaneously.

Supplementary Information The online version contains peer-reviewed but unedited supplementary material available at https://oi.org/10.1007/ s00125-021-05428-0.

Acknowledgements We gratefully acknowledge all participants for allowing the analysis of their genotypic and phenotypic data.

Data availability Type 1 diabetes summary statistics will be available through the GWAS catalogue (https://www.ebi.ac.uk/gwas/). Type 2 diabetes summary statistics are already publicly available.

Funding This work was funded by the JDRF (9-2011-253, 5-SRA-2015$130-\mathrm{A}-\mathrm{N})$ and Wellcome $(091157,107212)$ to the Diabetes and Inflammation Laboratory, University of Oxford. Additional funding 
was obtained from Wellcome $(090532,098381,106130,212259)$ and the National Institute of Diabetes and Digestive and Kidney diseases (U01DK105535). Computation used the Oxford Biomedical Research Computing (BMRC) facility, a joint development between the Wellcome Centre for Human Genetics and the Big Data Institute supported by Health Data Research UK and the NIHR Oxford Biomedical Research Centre. Financial support was provided by the Wellcome Trust Core Award Grant Number 203141/Z/16/Z. The views expressed are those of the author(s) and not necessarily those of the NHS, the NIHR or the Department of Health. Work was supported from grant U1301.2015/AI.1157.BE from Fondazione di Sardegna to Francesco Cucca.

Authors' relationships and activities MIM has served on advisory panels for Pfizer, Novo Nordisk and Zoe Global, has received honoraria from Merck, Pfizer, Novo Nordisk and Eli Lilly, and research funding from Abbvie, Astra Zeneca, Boehringer Ingelheim, Eli Lilly, Janssen, Merck, Novo Nordisk, Pfizer, Roche, Sanofi Aventis, Servier and Takeda. As of June 2019, MIM is an employee of Genentech and a holder of Roche stock. AM is an employee of Genentech since January 2020, and a holder of Roche stock. JRJI is an employee of Exploristics since June 2020. JAT serves on the advisory board of GSK. The remaining authors declare that there are no relationships or activities that might bias, or be perceived to bias, their work.

Contribution statement JRJI carried out the type 1 diabetes metaanalysis and the co-localisation analyses, drafted the manuscript and approved the final version. AM carried out the type 2 diabetes metaanalysis and conditional analyses, revised the article for intellectual content and approved the final version. CS and FC were involved in data collection in the Sardinia collection and carried out the association testing in this collection, revised the article for intellectual content and approved the final version. DJMC provided statistical advice and input, and made contributions to interpretation of the data, revised the article for intellectual content and approved the final version. MIS provided biological insight, contributed towards interpretation of the data, revised the article for intellectual content and approved the final version. MIM and JAT oversaw the research, contributed towards the conception, design and data collection, revised the article for intellectual content and approved the final version. JAT is the guarantor of this work.

Open Access This article is licensed under a Creative Commons Attribution 4.0 International License, which permits use, sharing, adaptation, distribution and reproduction in any medium or format, as long as you give appropriate credit to the original author(s) and the source, provide a link to the Creative Commons licence, and indicate if changes were made. The images or other third party material in this article are included in the article's Creative Commons licence, unless indicated otherwise in a credit line to the material. If material is not included in the article's Creative Commons licence and your intended use is not permitted by statutory regulation or exceeds the permitted use, you will need to obtain permission directly from the copyright holder. To view a copy of this licence, visit http://creativecommons.org/licenses/by/4.0/.

\section{References}

1. Onengut-Gumuscu S, Chen W-M, Burren O et al (2015) Fine mapping of type 1 diabetes susceptibility loci and evidence for colocalization of causal variants with lymphoid gene enhancers. Nat Genet 47:381-386

2. Mahajan A, Taliun D, Thurner M et al (2018) Fine-mapping type 2 diabetes loci to single-variant resolution using high-density imputation and islet-specific epigenome maps. Nat Genet 50:1505-1513

3. Aylward A, Chiou J, Okino M-L, Kadakia N, Gaulton KJ (2018) Shared genetic risk contributes to type 1 and type 2 diabetes etiology. Hum Mol Genet https://doi.org/10.1093/hmg/ddy314

4. McCarthy S, Das S, Kretzschmar W et al (2016) A reference panel of 64,976 haplotypes for genotype imputation. Nat Genet 48:1279 1283

5. Guo H, Fortune MD, Burren OS, Schofield E, Todd JA, Wallace C (2015) Integration of disease association and eQTL data using a Bayesian colocalisation approach highlights six candidate causal genes in immune-mediated diseases. Hum Mol Genet 24:33053313

6. Wallace C (2020) Eliciting priors and relaxing the single causal variant assumption in colocalisation analyses. PLoS Genet 16: e1008720. https://journals.plos.org/plosgenetics/article?id=10. 1371/journal.pgen. 1008720

7. Hormozdiari F, van de Bunt M, Segre AV et al (2016) Colocalization of GWAS and eQTL Signals Detects Target Genes. Am J Hum Genet 99:1245-1260

8. Vujkovic M, Keaton JM, Lynch JA et al (2019) Discovery of 318 novel loci for type-2 diabetes and related micro- and macrovascular outcomes among 1.4 million participants in a multi-ethnic metaanalysis. MedRxiv. https://doi.org/10.1101/19012690

9. Spracklen CN, Horikoshi M, Kim YJ et al (2020) Identification of type 2 diabetes loci in 433,540 East Asian individuals. Nature 582: 240-245. https://doi.org/10.1038/s41586-020-2263-3

10. Dooley J, Tian L, Schonefeldt S et al (2016) Genetic predisposition for beta cell fragility underlies type 1 and type 2 diabetes. Nat Genet 48:519-527

11. Eizirik DL, Pasquali L, Cnop M (2020) Pancreatic beta-cells in type 1 and type 2 diabetes mellitus: different pathways to failure. Nat Rev Endocrinol 16:349-362. https://doi.org/10.1038/s41574-0200355-7

Publisher's note Springer Nature remains neutral with regard to jurisdictional claims in published maps and institutional affiliations. 